\title{
CHAINS OF REINSURANCE REVISITED
}

\section{By Jean Lemaire and Jean-Pierre Quairiere}

\section{Université Libre de Bruxelles, Belgium}

\begin{abstract}
Chains of reinsurance were first modelled by Gerber, in a special case. It is shown that more general results can be obtained by applying Borch's theorem. The Pareto-optimal reinsurance indemnities are uniquely determined using the only assumption that the participating companies use exponential utility functions. A simple comparison then shows that Gerber's indemnities are not Pareto-optimal. Even if no assumption at all is introduced, the indemnities are shown to be closely linked to the risk aversions of the participants.
\end{abstract}

\section{KEYWORDS}

Reinsurance, risk exchange, bargaining theory.

\section{INTRODUCTION}

A chain of reinsurance $\left(C_{0}, C_{1}, \ldots, C_{n}\right)$ is a set of $n+1$ insurers, in which it is assumed that each participating company $C_{i}(i=1, \ldots, n-1)$ is in direct contact only with two others, namely $C_{1-1}$, to which coverage is sold, and $C_{i+1}$, from which coverage is bought. The last element of the chain, $C_{n}$, cannot buy any coverage, while the first element, $C_{0}$, cannot sell reinsurance. $C_{0}$, the ceding company, accepts a given risk in compensation of a premium $P_{0}$. The distribution function of the claims amount will be denoted by $G(x) . C_{l}$, in return of a premium $P_{1}$, accepts to pay an idemnity $I_{1}(x)$ to $C_{t-1}$, if the claims amount to $x$. In turn, $C_{t}$ buys coverage to $C_{t-1}$, in the form of an indemnity $I_{t+1}(x)$, and pays $P_{t+1}$ for this protection.

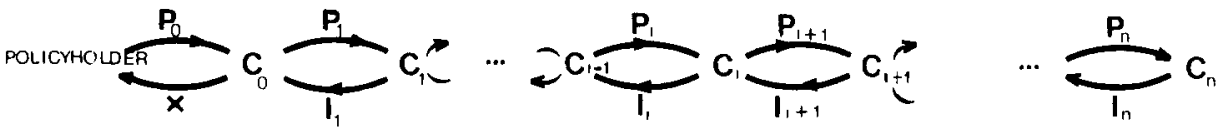

While chains of reinsurance are frequently encountered in practice, the first attempt to model mathematically their transactions is due to GERBER (1984), in a very special case:

-only proportional reinsurance is available;

-all companies apply the expected value principle;

-all companies evaluate their situation by means of an exponential utility function

$$
u_{i}(x)=\frac{1}{a_{1}}\left(1-e^{-a_{1} x}\right)
$$


- the claims are normally distributed;

- the following bargaining rules are enforced: the buyer of reinsurance lets the seller know how much he will buy as a function of the loading; the seller then determines the loading.

With those assumptions (some of which the practitioners will at the very least call heroic), it is possible to explicitly determine the loadings and the share of each participant, in a sequential way. In the special case $n=1$ (one reinsurance transaction only), the indemnity takes the very simple form

$$
I_{1}(x)=\frac{a_{0}}{2 a_{0}+a_{1}} x
$$

So the ceding company always retains at least $50 \%$ of the original claims, a counter-intuitive result. Cessions in excess of $50 \%$ are then shown to be possible, if the following (even more unrealistic) bargaining rules apply: the seller of reinsurance lets the buyer know how much he is willing to sell, as a function of the loading; the buyer then determines the loading on the basis of this information!

Gerber's results were extended by D'URSEL and LAUwERs (1985), with even stronger assumptions (equality of all risk aversion coefficients $a_{i}$, for instance), and criticized by BoRCH (1985).

The purpose of this paper is to show that more general results can be derived by applying the classical theorem of Borch. Indeed, the only assumption that the companies use exponential utility functions will allow us to determine explicitly the indemnities $I_{r}(x)$. In the special case $n=1$ we will obtain

$$
I_{1}(x)=\frac{a_{0}}{a_{0}+a_{1}} x,
$$

which shows that Gerber's indemnities are not Pareto-optimal. Then, the application of a value concept of game theory (for instance Nash's bargaining model) will enable us to find a unique set of premiums.

\section{BORCH'S RISK EXCHANGE MODEL}

This classical model (see for instance BuHLMANN 1970) considers a pool of $n$ insurance companies $C_{1}, \ldots, C_{n}$; the initial situation of $C_{j}$ is characterized by a pair $\left[R_{j}, G_{j}\left(x_{\jmath}\right)\right]$, where $R_{j}$ denotes its free reserves and $G_{j}\left(x_{j}\right)$ the distribution function of its claims amount. The members of the pool will try to improve their situations by concluding a treaty

$$
\bar{y}=\left[y_{1}\left(x_{1}, \ldots, x_{n}\right), \ldots, y_{n}\left(x_{1}, \ldots, x_{n}\right)\right],
$$

such that $\sum_{J=1}^{n} y_{J}\left(x_{1}, \ldots, x_{n}\right)=\sum_{J=1}^{n} x_{j} ; y_{j}\left(x_{1}, \ldots, x_{n}\right)$ is the sum $C_{j}$ has to pay if the claims for the different companies respectively amount to $x_{1}, \ldots, x_{n}$. The signature of such a treaty modifies the utility of $C_{j}$ from

$$
U_{j}\left(x_{\jmath}\right)=\int_{0}^{\infty} u_{j}\left(R_{\jmath}-x_{\jmath}\right) d G_{j}\left(x_{\jmath}\right)
$$


to

$U_{j}(\bar{y})$

$$
=\int_{\theta} u_{j}\left[R_{j}-y_{j}\left(x_{1}, \ldots, x_{n}\right)\right] d G\left(x_{1}, \ldots, x_{n}\right),
$$

where $\theta$ is the positive orthant of $E^{n}$ and $G\left(x_{1}, \ldots, x_{n}\right)$ the $n$-dimensional distribution function of the claims amounts $\vec{x}=\left(x_{1}, \ldots, x_{n}\right)$.

A treaty $\bar{y}$ is said to be Pareto-optimal if there is no $\bar{y}^{\prime}$ such that $U_{j}\left(\bar{y}^{\prime}\right) \geqslant U_{j}(\bar{y})$ for all $j$, with at least one strict inequality. Borch has shown that the set of the Pareto-optimal treaties is characterized by the equations

$$
k_{j} u_{j}^{\prime}\left[R_{j}-y_{j}(\bar{x})\right]=k_{1} u_{1}^{\prime}\left[R_{1}-y_{1}(\bar{x})\right], \quad j=1, \ldots, n,
$$

where the $k$, are non-negative bargaining constants. In the case of exponential utilities, the Pareto-optimal solutions take the form

$$
y_{j}(\bar{x})=q_{j} \sum_{i=1}^{n} x_{i}+y_{j}(0),
$$

where $q_{j}=\left(1 / a_{j}\right) /\left(\sum_{t=1}^{n} 1 / a_{i}\right)$, and $y_{j}(0)$ is a monetary transfer that depends on the bargaining constants. So a Pareto-optimal treaty is a quota share, where each company takes over a fraction of all claims inversely proportional to its risk aversion.

\section{FIRST MODEL}

In practice the reinsurance chain is formed by successive additions of companies: $C_{0}$ underwrites a risk and seeks coverage by $C_{1} ; C_{1}$ accepts part of the risk and contacts $C_{2}$, which in turn seeks protection by $C_{3}$, etc. Usually, a company $C_{1}$ will only be in touch with $C_{1+1}$ and $C_{1+1}$; it will be informed of the origin of the risk and of the cessions from $C_{0}$ to $C_{1-1}$; on the other hand, it will not be aware of the protective actions taken by $C_{t+1}$ (and hence of the continuation of the chain until $C_{n}$ ). $\mathrm{C}_{0}$, for instance, knows everything about its relations with $C_{1}$ but is usually totally unaware of the liabilities of $C_{2}, \ldots, C_{n}$ (just as an ordinary policyholder more often than not does not even know the name of his company's reinsurer). It is then quite realistic to model the chain as a set of $\boldsymbol{n}$ two-company treaties between $C_{t-1}$ and $C_{t}, i=1, \ldots, n$. The application of Borch's theorem to this specific risk exchange leads, for the treaty $i$, between $C_{i-1}$ and $C_{t}$, to the equation

$$
k_{\imath} u_{t}^{\prime}\left[R_{\imath}-y_{1}^{i}(x)\right]=k_{t-1} u_{t-1}^{\prime}\left[R_{t-1}-y_{t-1}^{l}(x)\right],
$$

(the upper indice denoting the treaty), where

$$
\begin{aligned}
y_{1}^{\prime}(x) & =I_{1}(x)-P_{1} \\
y_{t-1}^{\prime}(x) & =I_{t-1}(x)-P_{t-1}+P_{t}-I_{1}(x) .
\end{aligned}
$$

$k_{\imath}$ may be set equal to 1 . So

$$
u_{1}^{\prime}\left[R_{t}-I_{1}(x)+P_{1}\right]=k_{t-1} u_{\imath-1}^{\prime}\left[R_{t-1}-I_{t-1}(x)+I_{t}(x)+P_{\imath-1}-P_{\imath}\right] .
$$


The addition of the obvious conditions $I_{t}(0)=I_{t-1}(0)=0$ (no reinsurance payment if no claim) determines $k_{1-1}$

$$
k_{\imath-1}=\frac{u_{1}^{\prime}\left[R_{1}+P_{1}\right]}{u_{1-1}^{\prime}\left[R_{t-1}+P_{1-1}-P_{1}\right]} .
$$

Thus

$$
\frac{u_{t}^{\prime}\left[R_{t}-I_{t}(x)+P_{t}\right]}{u_{l}^{\prime}\left[R_{t}+P_{t}\right]}=\frac{u_{t-1}^{\prime}\left[R_{t-1}-I_{t-1}(x)+I_{t}(x)+P_{t-1}-P_{t}\right]}{u_{t-1}^{\prime}\left[R_{t-1}+P_{t-1}-P_{t}\right]} .
$$

Assuming exponential utilities, this equation becomes

$$
\frac{\exp \left(-a_{t}\left[R_{t}-I_{t}(x)+P_{t}\right]\right)}{\exp \left(-a_{t}\left[R_{t}+P_{t}\right]\right)}=\frac{\exp \left(-a_{t-1}\left[R_{t-1}-I_{t-1}(x)+I_{t}(x)+P_{t-1}-P_{t}\right]\right)}{\exp \left(-a_{t}\left[R_{t-1}+P_{t-1}-P_{t}\right]\right)}
$$

After easy computations, it simplifies to

$$
I_{i}(x)=\frac{a_{t-1}}{a_{t}+a_{t-1}} I_{t-1}(x), \quad i=1, \ldots, n .
$$

Each indemnity is a fraction of the preceding one: the risk progressively becomes diluted towards the end of the chain.

Since $I_{0}(x)=x$, successive replacements lead to

$$
I,(x)=\left(\prod_{j=1}^{1} \frac{a_{j-1}}{a_{j}+a_{j-1}}\right) x, \quad i=1, \ldots, n .
$$

Each indemnity is a fraction of the claim amount, that depends on all the "preceding" risk aversion coefficients, but is independent of $a_{i+1}, a_{t+2}, \ldots, a_{n}$. A consequence is that the addition of a supplementary link $C_{n+1}$ to the chain does not modify the indemnities.

$I_{1}(x)$ is a decreasing function of $a_{1}$ : the highly risk-averse companies will accept only small liabilities; the largest part of the risk is transferred to the companies with a low degree of risk aversion. The limiting case $a_{1}=0$ means that $C_{1}$ is a risk neutral company that does not buy any reinsurance: $I_{1}(x)=I_{1-1}(x) ; I_{1+1}(x)=$ 0 ; the chain does not extend beyond $C_{l}$. The other limiting case $a_{1}=\infty$ of extreme risk aversion leads to $I_{l}(x)=0: C_{1}$ refuses to accept liability and acts as a broker.

$I_{1}(x)$, the payment of $C_{1}$ to $C_{1-1}$, is only a "gross" disbursement for $C_{1}$, since it collects $I_{t+1}(x)$ from $C_{t+1}$.

The "net" payment

$$
D_{\imath}(x)=I_{\imath}(x)-I_{\imath+1}(x)
$$

is equal, after replacement, to

$$
D_{\imath}(x)=\left(\prod_{j=1}^{1} \frac{a_{J-1}}{a_{j-1}+a_{j}}\right) \cdot \frac{a_{t+1}}{a_{t}+a_{t+1}} \cdot x .
$$

So the Pareto-optimal treaties are proportional; $C_{i}$ 's share is a function of all risk aversion coefficients from $a_{0}$ to $a_{i+1}$; the quotas are independent of the claims amount distribution and do not have to be negotiated. 
It is easy to check that

$$
D_{i}(x)=\frac{a_{\imath-1}}{a_{t}} \frac{a_{i+1}}{a_{i}+a_{i+1}} D_{\imath-1}(x)
$$

So, if $a_{i-1}<a_{1}, D_{1}(x)<D_{1-1}(x)$.

It has been shown already that the addition of a last link to the chain does not modify the indemnities. What happens if a new company, $C_{k}$, is inserted between $C_{1-1}$ and $C_{1}$ ? Clearly the indemnities $I_{j}(x), j=1, \ldots, i-1$ and the net payments $D_{j}(x), j=1, \ldots, i-2$ are not modified. For $l \geqslant i$, the new indemnity equals

$$
I_{l}^{\prime}(x)=\left(\prod_{\substack{j=1 \\ j \neq 1}}^{l} \frac{a_{j-1}}{a_{j-1}+a_{j}}\right) \cdot \frac{a_{t-1}}{a_{t-1}+a_{k}} \cdot \frac{a_{k}}{a_{k}+a_{i}} \cdot x .
$$

The modified net payment $D_{l}^{\prime}(x)$ equals

$$
D_{l}^{\prime}(x)=\left(\prod_{\substack{j=1 \\ j \neq 1}}^{i} \frac{a_{j-1}}{a_{j-1}+a_{j}}\right) \cdot \frac{a_{1-1}}{a_{t-1}+a_{k}} \cdot \frac{a_{k}}{a_{k}+a_{l}} \cdot \frac{a_{l}}{a_{l}+a_{l+1}} \cdot x .
$$

Simple computations lead to

$$
D_{l}(x)-D_{l}^{\prime}(x)=\left(\prod_{\substack{j=1 \\ j \neq 1}}^{l} \frac{a_{j-1}}{a_{j-1}+a_{j}}\right) \cdot \frac{a_{l+1}}{a_{l}+a_{i+1}}\left[\frac{a_{t-1}}{a_{t-1}+a_{\imath}}-\frac{a_{t-1}}{a_{t-1}+a_{k}} \frac{a_{k}}{a_{k}+a_{i}}\right] \cdot x
$$

which is always positive. So the insertion of a supplementary link always has the effect of decreasing the net payments of all the companies "following" the new one.

It is important to notice that in this model we only have "local" Paretooptimality, since, while applying Borch's theorem to e.g. $C_{t-1}$ and $C_{l}$, it is deliberately ignored that $C_{\imath}$ will subsequently seek reinsurance by $C_{\imath+1}$. While it is not infrequent in practice to accept a risk before seeking protection, in many cases existing treaties certainly influence the negotiation between two links of a chain. Presumably the risk aversion coefficient of a company will strongly depend on whether reinsurance is already available or not. Note that a "global" Paretooptimal model is presented in Section 7.

\section{PREMIUM DETERMINATION}

As shown in the preceding section, the shares of the participants in each claim are uniquely determined; they are not subject to bargaining (this is a well-known property of exponential utilities). The fact that the Pareto-optimal solution set generally consists of an infinity of treaties is reflected in the indefiniteness of the premiums $P_{i}$; there usually exists an infinity of acceptable $P_{l}$, even if the individual rationality condition (no company will accept a decrease in utility) is enforced. In fact, the determination of the premium $P_{1}$ is equivalent to the computation of the value of a two-person cooperative game without transferable utilities between $C_{1}$ and $C_{t-1}$ (see for instance Lemaire 1979). 
As an illustration, let us compute the Nash value of the chain (NASH 1950). Nash has shown that one and only one treaty satisfies the four following axioms:

1. Pareto-optimality

2. Linear invariance

The solution is not affected by a linear transformation performed on the players' utilities.

3. Symmetry

Every symmetric game has a symmetric solution; if the players are not distinguishable by the rules of the game, the value shall yield them equal utility payoffs.

4. Independence of irrelevant alternatives

The solution is not affected by removal, from the feasible set, of any point except the solution itself and the disagreement point (no reinsurance, in our case).

The only treaty that satisfies those axioms is obtained by maximizing the product of the players' utility gains. In our model, this leads, for the negotiation of $P_{1}$ between $C_{i}$ and $C_{t-1}$, to

$$
\begin{aligned}
\max _{P_{t}}\left\{U_{t-1}\left[R_{t-1}-I_{t-1}(x)+I_{t}(x)+P_{t-1}-P_{t}\right]-U_{t-1}\left[R_{t-1}+P_{t-1}-I_{t-1}(x)\right]\right\} \\
\quad \times\left\{U_{t}\left[R_{t}-I_{t}(x)+P_{t}\right]-U_{t}\left(R_{t}\right)\right\} \\
=\max _{P_{t}}\left\{E\left[\frac{1}{a_{t-1}}\left(1-\exp \left[-a_{t-1}\left(R_{t-1}-I_{t-1}(x)+I_{t}(x)+P_{t-1}-P_{t}\right)\right]\right)\right]\right. \\
\left.-E\left[\frac{1}{a_{t-1}}\left(1-\exp \left[-a_{t-1}\left(R_{t-1}-I_{t-1}(x)+P_{t-1}\right)\right]\right)\right]\right\} \\
\times\left\{E\left[\frac{1}{a_{t}}\left(1-\exp \left[-a_{\imath}\left(R_{t}-I_{t}(x)+P_{t}\right)\right]\right)\right]-\frac{1}{a_{t}}\left(1-e^{-a_{t} R_{t}}\right)\right\} .
\end{aligned}
$$

It is equivalent to maximize

$$
\begin{aligned}
& \left\{-e^{a_{t-1} P_{i}} E\left\{\exp \left[-a_{i-1}\left(I_{t}(x)-I_{i-1}(x)\right)\right]\right\}+E\left[\exp \left(a_{t-1} I_{t-1}(x)\right)\right]\right\} \\
& \quad \times\left\{1-\exp \left(-a_{i} P_{t}\right) E\left[\exp \left(a_{i} I_{i}(x)\right)\right]\right\}
\end{aligned}
$$

Denote $M(t)$ the moment-generating function of the claims amount distribution, and let

$$
\begin{aligned}
& A_{\imath}=E\left\{\exp \left[-a_{t-1}\left(I_{t}(x)-I_{t-1}(x)\right)\right]\right\}=M\left[a_{t-1}\left(i_{t-1}-i_{t}\right)\right] \\
& B_{\imath}=E\left[\exp \left(a_{\imath} I_{\imath}(x)\right)\right]=M\left[a_{\imath} i_{\imath}\right] \\
& C_{\imath}=E\left[\exp \left(a_{t-1} I_{t-1}(x)\right)\right]=M\left[a_{t-1} i_{t-1}\right],
\end{aligned}
$$

introducing the notation $i_{l}=I_{l}(x) / x$. The product to maximize reduces to

$$
\left[-A_{1} \cdot e^{a_{t-1} P_{t}}+C_{1}\right]\left[1-B_{1} \cdot e^{-a_{t} P_{t}}\right] .
$$


The first order condition is

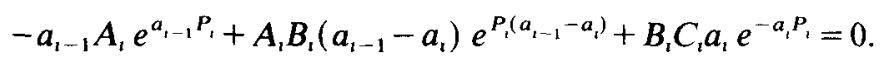

Let

$$
\begin{aligned}
& A=-a_{t-1} A_{t} \\
& B=A_{t} B_{t}\left(a_{t-1}-a_{t}\right) \\
& C=a_{1} B_{t} C_{t} \\
& X=e^{a_{t-1} P_{t}} \\
& Y=e^{-a_{1} P_{t}}
\end{aligned}
$$

The equation reduces to

$$
A X+B X Y+C Y=0 .
$$

This is the equation of a conic, of center $(-C / B,-A / B)$, if $B \neq 0$. By performing the change of variables

$$
X^{\prime \prime}=X+\frac{C}{B} \quad \text { and } \quad Y^{\prime}=Y+\frac{A}{B},
$$

the equation becomes

$$
X^{\prime} Y^{\prime}=\frac{A C}{B^{2}}
$$

and can easily be solved numerically.

If $B=0$, the equation reduces to $A X=-C Y$, and the solution is

$$
P_{1}=\frac{\log (-C / A)}{a_{1-1}+a_{1}}
$$

Nash's solution possesses numerous interesting properties (ROTH 1979). Among others, the utility assigned to a player increases as his opponent becomes more risk-averse. So the higher the risk aversion of a participant of the chain, the higher the premium $P_{t}$ he will pay for his protection.

A first approximation of $P_{1}$ can be obtained from equation (3) by expanding the exponentials around the reserves. The first-order approximation is

$$
\frac{1+a_{t}\left[I_{t}(x)-P_{t}\right]}{1-a_{t} P_{t}}=\frac{1+a_{t-1}\left[P_{t}-P_{t-1}+I_{1-1}(x)-I_{t}(x)\right]}{1+a_{t-1}\left(P_{t}-P_{t-1}\right)} \text {. }
$$

A straightforward development, using relation

$$
\frac{I_{t}(x)}{I_{t-1}(x)}=\frac{a_{t-1}}{a_{t-1}+a_{t}},
$$

leads to

$$
P_{1}=\frac{a_{t-1}}{a_{1}+a_{t+1}} P_{t-1}
$$


and to

$$
P_{1}=\left(\prod_{j=1}^{1} \frac{a_{j-1}}{a_{j-1}+a_{j}}\right) P_{0} .
$$

So, as a first approximation, the same relationship applies to the indemnities and the premiums: any participant in the chain will pay a certain fraction of all claims, after collecting the same fraction of the original premium.

\section{COMPARISON WITH GERBER'S MODEL}

Applied to the simple transaction between an insurance company and its reinsurer $(n=1)$, formula $(4)$ reduces to

$$
I_{1}(x)=\frac{a_{0}}{a_{0}+a_{1}} x
$$

while Gerber's model led to

$$
I_{1}^{\prime}(x)=\frac{a_{0}}{2 a_{0}+a_{1}} x
$$

Consequently the latter proposal is not Pareto-optimal.* This can be shown directly. Denote $U_{0}$ and $U_{1}$ the expected utilities when the indemnity is $I_{1}(x)$, and $U_{0}^{\prime}$ and $U_{1}^{\prime}$, the expected utilities corresponding to $I_{1}^{\prime}(x)$; we need to show that

$$
U_{0} \geqslant U_{0}^{\prime} \text { and } U_{1} \geqslant U_{1}^{\prime}, \quad \text { with at least one strict inequality. }
$$

The first inequality

$$
\begin{aligned}
\int_{0}^{\infty} \frac{1}{a_{0}}\left[1-\exp \left(-a_{0}\left[R_{0}+P_{0}-x-P_{1}+I_{1}(x)\right]\right)\right] d G(x) \\
\geqslant \int_{0}^{\infty} \frac{1}{a_{0}}\left[1-\exp \left(-a_{0}\left[R_{0}+P_{0}-x-P_{1}^{\prime}+I_{1}^{\prime}(x)\right]\right)\right] d G(x)
\end{aligned}
$$

reduces to

$$
e^{a_{0} P_{1}} M\left[a_{0}\left(1-i_{1}\right)\right] \leqslant e^{a_{0} P_{i}^{\prime}} M\left[a_{0}\left(1-i_{1}^{\prime}\right)\right] .
$$

If the claim amounts are normally distributed, of mean $m$ and standard deviation $\sigma^{2}, M(t)=e^{t m} e^{t^{2} \sigma^{2} / 2}$ and the inequality becomes

$$
P_{1}+\left(1-i_{1}\right) m+\frac{a_{0}\left(1-i_{1}\right)^{2} \sigma^{2}}{2} \leqslant P_{1}^{\prime}-\left(1-i_{1}^{\prime}\right) m+\frac{a_{0}\left(1-i_{1}^{\prime}\right)^{2} \sigma^{2}}{2} .
$$

The same computations for the second inequality lead to

$$
-P_{1}+m i_{1}+\frac{a_{1} i_{1}^{2} \sigma^{2}}{2} \geqslant-P_{1}^{\prime}+i_{1}^{\prime} m+\frac{\sigma^{2} a_{1} i_{1}^{\prime 2}}{2}
$$

* Of course, in a two-company chain, "local" and "global" Pareto-optimality amount to the same thing. 
Assembling the two inequalities, we obtain

$$
P_{1}^{\prime}+m\left(i_{1}-i_{1}^{\prime}\right)+\frac{a_{1} \sigma^{2}}{2}\left(i_{1}^{2}-i_{1}^{\prime 2}\right) \leqslant P_{1} \leqslant P_{1}^{\prime}+m\left(i_{1}-i_{1}^{\prime}\right)+\frac{a_{0} \sigma^{2}}{2}\left[\left(1-i_{1}^{\prime}\right)^{2}-\left(1-i_{1}\right)^{2}\right] \text {. }
$$

So, whatever the premium $P_{1}^{\prime}$ in Gerber's model, it is possible to find a premium $P_{1}$ in our model that improves both companies' situations iff

$$
a_{1}\left(i_{1}^{2}-i_{1}^{\prime 2}\right)<a_{0}\left[\left(1-i_{1}^{\prime}\right)^{2}-\left(1-i_{1}\right)^{2}\right]
$$

Replacing $i_{1}$ and $i_{1}^{\prime}$ by their respective values $a_{0} /\left(a_{0}+a_{1}\right)$ and $a_{0} /\left(2 a_{0}+a_{1}\right)$, it is easily seen that this inequality is always satisfied.

\section{OTHER UTILITY FUNCTIONS}

Some results can be obtained without specifying the form of the utility function. According to the Taylor-Lagrange theorem there exists $0<\theta<1$ and $0<\delta<1$ such that (1) can be written

$$
\begin{aligned}
& \frac{u_{t}^{\prime}\left(R_{t}+P_{\imath}\right)-I_{t}(x) u_{t}^{\prime \prime}\left(R_{t}+P_{t}-\theta I_{t}(x)\right)}{u_{\imath}^{\prime}\left(R_{t}+P_{t}\right)} \\
& =\frac{u_{\imath-1}^{\prime}\left(R_{t-1}+P_{t-1}-P_{\imath}\right)+\left[I_{1}(x)-I_{t-1}(x)\right] u_{t-1}^{\prime \prime}\left[R_{t-1}+P_{t-1}-P_{t}+\delta\left(I_{t}(x)-I_{t-1}(x)\right)\right]}{u_{t-1}^{\prime}\left(R_{t-1}+P_{t-1}-P_{\imath}\right)} \text {. }
\end{aligned}
$$

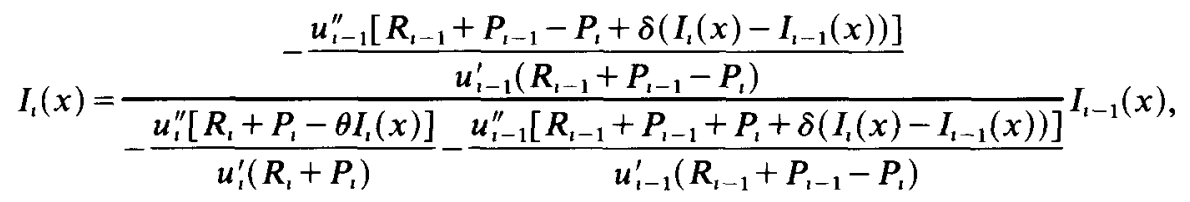

$$
\begin{aligned}
& I_{l}(x) \approx \frac{r_{t-1}\left(R_{t-1}+P_{t-1}-P_{\imath}\right)}{r_{l}\left(R_{l}+P_{\imath}\right)+r_{t-1}\left(R_{t-1}+P_{t-1}-P_{\imath}\right)} I_{t-1}(x)
\end{aligned}
$$

where $r_{\imath}(x)=-u_{1}^{\prime \prime}(x) / u_{\imath}^{\prime}(x)$ classically denotes the risk aversion function of $C_{\imath}$. So a highly risk averse company will only take over a small portion of the liabilities of its predecessor in the chain.

By differentiating (2) with respect to $x$, we obtain

$$
\begin{aligned}
& \frac{u_{t}^{\prime \prime}\left[R_{t}+P_{t}-I_{t}(x)\right]}{u_{1}^{\prime}\left(R_{t}+P_{t}\right)}\left(-\frac{\partial I_{t}(x)}{\partial x}\right)=\frac{u_{t-1}^{\prime \prime}\left[R_{t-1}+P_{t-1}-P_{t}+I_{t}(x)-I_{t-1}(x)\right]}{u_{t-1}^{\prime}\left(R_{t-1}+P_{t-1}-P_{t}\right)} \times\left(\frac{\partial I_{t}(x)}{\partial x}-\frac{\partial I_{t-1}(x)}{\partial x}\right) \\
& \frac{\partial I_{t}(x)}{\partial x}=\frac{\frac{u_{t-1}^{\prime \prime}\left[R_{t-1}+P_{t-1}-P_{t}+I_{t}(x)-I_{t-1}(x)\right]}{u_{t-1}^{\prime}\left(R_{t-1}+P_{t-1}-P_{t}\right)}}{\frac{u_{t-1}^{\prime \prime}\left[R_{t-1}+P_{t-1}-P_{t}+I_{t}(x)-I_{t-1}(x)\right]}{u_{t-1}^{\prime}\left(R_{t-1}+P_{t-1}-P_{t}\right)}+\frac{u_{1}^{\prime \prime}\left[R_{t}+P_{t}-I_{t}(x)\right]}{u_{t}^{\prime}\left(R_{t}+P_{t}\right)} \frac{\partial I_{t-1}(x)}{\partial x} .}
\end{aligned}
$$


So, except in the case of risk neutrality,

$$
\frac{\partial I_{i}(x)}{\partial x}<\frac{\partial I_{1-1}(x)}{\partial x}
$$

the risk becomes more and more diluted as the chain progresses; the indemnities become less sensitive to the claims.

\section{SECOND MODEL}

In our second model we assume that the whole chain is entirely formed, and known by all participants, when the risk is underwritten. A "global" Paretooptimal treaty is sought; Borch's theorem has to be applied simultaneously to all companies

$$
u_{\imath}^{\prime}\left[R_{\imath}-y_{\imath}(x)\right]=k_{t-1} u_{i-1}^{\prime}\left[R_{t-1}-y_{\imath-1}(x)\right], \quad i=1, \ldots, n,
$$

with

$$
y_{t-1}(x)=P_{1}-P_{t-1}+I_{t-1}(x)-I_{1}(x)
$$

and

$$
y_{\mathrm{t}}(x)=P_{\mathrm{t}+1}-P_{\mathrm{t}}+I_{\mathrm{t}}(x)-I_{\mathrm{t}+1}(x) \text {. }
$$

We obtain, again assuming exponential utilities,

$$
\begin{aligned}
\exp ( & \left.-a_{1}\left[R_{t}-P_{t+1}+P_{t}-I_{t}(x)+I_{t+1}(x)\right]\right) \\
& =k_{t-1} \exp \left(-a_{t-1}\left[R_{t-1}-P_{t}+P_{t-1}-I_{\imath-1}(x)+I_{t}(x)\right]\right) .
\end{aligned}
$$

If $x=0, I_{i}(x)=I_{i-1}(x)=I_{i+1}(x)=0$, and

$$
k_{l-1}=\exp \left[-a_{\imath}\left(R_{t}-P_{\imath+1}+P_{\imath}\right)\right] / \exp \left[-a_{\imath-1}\left(R_{\imath-1}-P_{\imath}+P_{t-1}\right)\right] .
$$

Then

$$
\begin{aligned}
\frac{\exp \left(-a_{t}\left[R_{t}-P_{t+1}+P_{t}-I_{t}(x)+I_{t+1}(x)\right]\right)}{\exp \left(-a_{t}\left[R_{t}-P_{t+1}+P_{t}\right]\right)} \\
=\frac{\exp \left(-a_{t-1}\left[R_{t-1}-P_{t}+P_{t-1}-I_{t-1}(x)+I_{t}(x)\right]\right)}{\exp \left(-a_{t-1}\left[R_{t-1}-P_{t}+P_{t-1}\right]\right)} .
\end{aligned}
$$

After simplifications, we obtain the following system of $n$ equations with $n$ unknowns

$$
-a_{t-1} I_{t-1}(x)+\left(a_{t-1}+a_{i}\right) I_{1}(x)-a_{1} I_{1+1}(x)=0, \quad i=1, \ldots, n
$$

with $I_{0}(x)=x, I_{n+1}(x)=0$.

So

$$
a_{1}\left[I_{1}(x)-I_{t+1}(x)\right]=a_{t-1}\left[I_{t-1}(x)-I_{t}(x)\right] \text { for all } i=1, \ldots, n \text {. }
$$


Denote this common quantity by $H$. Since $I_{0}(x)=x$ and $I_{n+1}(x)=0$, we have immediately

$$
x=\sum_{i=0}^{n} \frac{H}{a_{i}}
$$

and

$$
H=x / \sum_{i=0}^{n} \frac{1}{a_{i}} .
$$

Thus the net payment of company $i$ is

$$
I_{i}(x)-I_{i+1}(x)=\frac{1}{a_{i}} / \sum_{j=0}^{n} \frac{1}{a_{j}} \cdot x
$$

The gross payment is

$$
I_{i}(x)=\sum_{k=1}^{n} \frac{1}{a_{k}} / \sum_{j=0}^{n} \frac{1}{a_{j}} \cdot x, \quad i=0, \ldots, n .
$$

The determination of the premiums is now more complicated, since it is equivalent to the computation of the value of a $(n+1)$-person cooperative game without transferable utilities. Several value concepts are suitable, but the calculations become extremely complex. A first approximation of $P_{1}$ can be obtained, like in the first model, by expanding the exponentials in (5) around the reserves. The first-order approximation is

$$
\frac{1+a_{1}\left[P_{t+1}-P_{1}+I_{t}(x)-I_{t+1}(x)\right]}{1+a_{1}\left(P_{t+1}-P_{1}\right)}=\frac{1+a_{t-1}\left[P_{t}-P_{t-1}+I_{t-1}(x)-I_{1}(x)\right]}{1+a_{1-1}\left(P_{1}-P_{t-1}\right)} .
$$

After development and repeated use of (6) this reduces to

$$
-a_{i-1} P_{i-1}+\left(a_{i}+a_{1-1}\right) P_{1}-a_{i} P_{i+1}=0 .
$$

The same relationship thus applies to the premiums and the indemnities. Consequently, in first approximation,

$$
P_{t}=\sum_{k=1}^{n} \frac{1}{a_{k}} / \sum_{j=0}^{n} \frac{1}{a_{j}} \cdot x:
$$

the same fraction is to be applied to the initial premium and to the claims to obtain the liabilities of each participant.

Note that formula (7) is nothing else than (1): each company pays a net share of each claim which is inversely proportional to its risk aversion. So the chain reduces to the "classical" risk exchange.

\section{ACKNOWLEDGMENTS}

The authors wish to thank the referees for valuable suggestions that considerably improved the paper. 


\section{REFERENCES}

BorCH, K. (1985) Some Comments on the Paper: Chains of Reinsurance: Non-Cooperative Equilibria and Pareto-Optimality. 12th Seminar of the European Group of Insurance Economics, Geneva Association.

BühlmanN, H. (1970) Mathematical Methods in Risk Theory. Springer-Verlag: Berlin.

GERBER, H. (1984) Chains of Reinsurance. Insurance: Mathematics and Economics, 3, 43-48.

Lemaire, J. (1979) Reinsurance as a Cooperative Game. In Applied Game Theory, Physica-Verlag, Würzburg, 254-269.

Nash, J. (1950) The Bargaining Problem. Econometrica, 18 155-162.

Jean Lemaire and Jean-Pierre Quairiere

Université Libre de Bruxelles, Institut de Statistique, C.P. 210, 50 Boulevard du Triomphe B-1050 Bruxelles. 